\title{
EQUATION OF STATE AND IONIZATION OF DENSE PLASMAS
}

\author{
W. EBELING \\ Humboldt-Universität \\ Sektion Physik \\ 1040 Berlin \\ DDR
}

ABSTRACT. In large regions of densities and temperatures the properties of plasmas are influenced by Coulombic forces. Here especially hydrogen and helium plasmas are studied. The region of nonideality is given and the main effects as screening, quantum corrections, level shifts and the cut off for the sum over states are discussed. Based on methods of classical and quantum statistics corrections to the ideal EOS are calculated. Interpolational expressions covering the whole densitytemperature plane are given and phase transitions to highly ionized states are discussed. Level shifts due to nonideality are derived. Finally the ionization equilibrium and kinetics are considered theoretically focussing the attention on nonideality effects.

\section{Introduction}

From the point of view of plasma physics the sun is a big plasma ball which might be considered as a giant laboratory realizing plasmas in a broad range of state parameters. For example we meet dense plasmas with more than 1025 particles $/ \mathrm{cm}^{3}$ in the center where the temperature is above $10^{7} \mathrm{~K}$ and on the other hand we find very diluted plasmas in the outer regions with temperatures below $10^{4} \mathrm{~K}$. For our crude physicists approach we model the sun as a spherical plasma consisting of about 90-93\% hydrogen, 6-8\% Helium and less 
than $2 \%$ heavier particles, (all numbers refer to mol percents). We consider the plasma ball as being in mechanical equilibrium as well as in thermodynamical equilibrium with given distributions of temperature and densities along the radius. Hydrodynamic flows and other transport processes expect radiation will be neglected. However strong non-equilibrium with respect to nuclear and ionization reactions and with respect to radiating transitions is to be taken into account From the physicists point of view the radiation from the sun which is absorbed and reemitted by the earth, constitutes the driving force of all selforganization processes on our planet [1]. A mechanism which we have called the photon mill [2] provides the earth with the export of entropy which is the necessary condition for the evolution of life, man, science and culture. We can easily estimate the flow of entropy from the system sunearth to the sea of background radiation by assuming that in average $230 \mathrm{~W} / \mathrm{m}^{2}$ are absorbed on the surface in the earth and are reemitted. Assuming that the temperature of the incoming photons is about $5800 \mathrm{~K}$ and that the earth radiates as a black body of $260 \mathrm{~K}$ we get the entropy export flow

$$
J_{s}=\frac{4}{3} \cdot\left(\frac{230}{5800}-\frac{230}{260}\right) \frac{W}{m^{2} K} \approx-1 \frac{W}{m^{2} K}
$$

It is quite sure that any essential change of these numbers would have dramatic consequences for the conditions of life on our planet. the fundamental role of the sun for our life is a strong motivation for an increasing number of physicists, to provide the astrophysicists with better tools for an investigation of the basic physical processes in the sun. Here we restrict our consideration to a few physical properties and processes which are of relevance in this respect. We will start with a review of several methods of statistical thermodynamics and will develope then a new variant of the theory including an occupation number formalism which seems to be appropriate for the study of the sun. The idea of this approach is that each (internal) state of bound particles (atoms, molecules and ions) is treated as a separate species characterized by specific properties as e.g. an effective volume and by a relative occupation number. A similar approach was developed by 
Rogers [3] and is being used now for extended calculations of the opacity of the sun [4] and of the EOS of the solar interior [5]. Another closely related approach was developed by Hummer, Mihalas, Däppen and others [6]. In difference to Rogers activity expansion method, our approach is based on a density expansion (canonical ensemble) $[6,7]$ which has the advantage that generalizations to kinetic processes are more easy. Another advantage is the good convergence of density expansions in comparison to fugacity expansions $[8,9]$.

\section{Parameter regions and survey of theoretical methods}

Let us consider a plasma consisting of $\mathrm{n}_{\mathrm{e}}$ electrons and nuclei or ions with positive charges $z_{i}$.e $(i=\ldots$ s) per unit volume. We define the total number of positive charges and charge averages by

$$
n_{+}=\sum_{i} n_{i} ;\left\langle z^{p}\right\rangle=n_{+}^{-1} \sum_{i} z_{i}^{p} n_{i}
$$

By introducing the mean distance between electrons and positive charges

$$
d_{e}=\left(\frac{3}{4} \pi n_{e}\right)^{\frac{1}{3}} ; d_{+}=\left(\frac{3}{4} \pi n_{+}\right)^{\frac{1}{3}}
$$

we define now two dimensionless correlation parameter

$$
\Gamma_{e}=\frac{e^{2}}{\theta_{e} D_{e}} ; \Gamma_{+}=\frac{e^{2}}{\theta_{+} D_{+}}\left\langle z^{5 / 3} y_{z}\right\rangle^{1 / 3}
$$

Here $\theta_{\mathrm{e}}$ and $\theta_{+}$are average kinetic energies which include degeneracy; in the classical case we have simply $\theta_{e}=\theta_{+}=$ $\mathrm{k}_{\mathrm{B}} \mathrm{T}$. The curves $\Gamma_{\mathrm{e}}=1, \Gamma_{+}=1$ separate the regions of strong and weak correlations respectively. In addition we define a parameter which describes the strength of the electron-ion interactions by

$$
\xi=2(z)\left(\frac{I}{\theta_{e}}\right)^{1 / 2} ; \theta_{e}=\frac{p_{e}^{i d}}{n_{e}}
$$


where $I$ is the ionization energy of hydrogen. Inside the line $\xi=1$ we observe strong interactions e.g. in scattering and bound state effects. Typical parameters of the sun are

$$
\Gamma_{+} \leq .1 ; 0.01 \leq \xi \leq 100
$$

This shows that the matter in the sun is in conditions of weak nonideality but strong two-particle interactions. Another important parameter is the relative accupation of the available space by bound states

$$
\eta=\frac{4 \pi}{3} \sum_{b} n_{b} R_{b}^{3}
$$

where $n b$ and $R b$ are the density and the effective radius of the bound stable $b$. In the region $\eta \geq 1$ bound states break down due to the strong Pauli blocking effects which make bound states extremely unfavorable in the thermody namic sense.

The existing theories [8-17] are mostly restricted to special regions in the density-temperatures plane. In the region of strong interactions $\xi \geq 10$ where atoms and molecules dominate, one of the most appropriate theories is the fluid variational theory [10]. In this approach one starts from the following decomposition of the free energy density

$$
f=f_{I D}+f_{H C}+f_{V W}+f_{C O}
$$

The coulombic part is zero in the region, where only neutrals exist. The hard-core part is approximated by standard expressions

$$
f_{H C}=k_{B} T \frac{4 \eta-3 \eta^{2}}{\left(1-\eta^{2}\right)^{2}}+\text { corrections }
$$

The Van der Waals part is represented by perturbation theory

$$
f_{v w}=4 \pi n^{2} \int_{R}^{\infty} \Phi(r) g_{H S}(r, \eta) r^{2} d r
$$


Here $\Phi(r)$ is the effective interaction potentiel for the neutrals, a good choice seems to be exponential-sixpotential $[10,11]$

$$
\begin{gathered}
\Phi(r)=\varepsilon\left\{\frac{6}{\alpha-6} \exp \left[\alpha \frac{r_{m}-r}{r_{m}}\right]-\frac{\alpha}{\alpha-6}\left(\frac{r_{m}}{r}\right)^{6}\right\} \\
H_{2}, D_{2}: \varepsilon / k_{B}=36.4 \mathrm{~K} ; r_{m}=3.49 \mathrm{~A} ; \alpha=11.1 \\
H_{e}: \quad \varepsilon / k_{B}=10.8 \mathrm{~K} ; r_{m}=2.97 \AA ; \alpha=13.1
\end{gathered}
$$

Further $g_{++s}(r)$ is the radial correlation function for hard spheres. The radius $R$ is considered as a variational parameter of the theory. In real plasmas we have often several neutral species and therefore more elaborate theories for mixture of hard spheres with different diameters have to be used. In our calculations we have used the expressions developed by Mansouri [12] in combination with simple approximations for the attracting forces $[18,19]$

$$
\begin{gathered}
f_{H C}=f_{M A N S} ; f_{V D W}=-\sum n_{i} n_{j} A_{i j} \\
A_{i j}=-4 \pi \int_{R_{i}+R_{j}} \phi_{i j}(r) r^{2} d r
\end{gathered}
$$

Let us still note, that in this approach electrons and nuclei have to be considered as spheres with zero radius $[18,19]$.

Another limiting case of great importance is the classical Coulomb fluid consisting of heavy charged particles (protons, deutrons and higher charged nuclei) imbedded into a neutralizing uniform sea of electrons. For this special model many data from Monte Carlo studies are available $[14,15]$. The free energy density may approximated by the analytical formula for the free energy density

$$
f_{C O}=-n_{+} k_{B} T\left(\beta e^{2}\right)^{\beta} 1.447\left(z^{5 / 3}\right)(z)^{1 / 3}
$$

Instead of this simple expression more elaborate formulae may be used $[13 \cdot 15,18,19]$.

The methods described so far are restricted to special regions of the temperature-density plane. A more universal method is the Green functions approach developed by Martin, Schwinger, 
Kadanoff, Baym, Abrikossov and others. In this method thermodynamic functions follow from the relation between density $n$, chemical potential and one particle Green function [7]

$$
n(\mu)=V^{-1} \sum_{p} \int \frac{d \omega_{f}}{2 \pi} f_{1}(\omega) I m G_{1}(p, \omega)
$$

The Green function is expressed by Dysons equation

$$
G_{1}=G_{1}^{0}+G_{1}^{0} \Sigma_{1} G_{1}
$$

where $G_{1}^{0}$ is the Green function for free particles and $\Sigma_{1}$ is the self-energy which may be represented as an infinitive sum of diagrams. Within all these contributions we need a guidding idea to select out the relevant diagrams. For this we developed a special method, an occupation number formalism based on the "chemical picture" [7-9].

This may be described in short as follows: we approximate the selfenergy by ladder type diagrams

$$
\Sigma_{1}^{L} G_{1}^{0}=V G_{2}^{L}
$$

All diagrams containing free particle propagators are completed by diagrams containing a propagator for the composites [16]. With these approximations for $G_{1}$ we arrive at expressions for the thermodynamic functions which contain also higher order correlations and n-particle composites

$$
n(\mu)=\sum_{p} f_{1}\left(\varepsilon_{1}\right)+\sum_{k P} g_{2}\left(E_{k P}\right)+\sum_{p} \int d \omega g_{2}(\omega) \frac{d \sin \delta(\omega)}{d \omega}
$$

where $\delta(\omega)$ is a generalized scattering phase. The energy levels contain first order perturbation shifts with free and bound state contributions

$$
\begin{gathered}
\varepsilon_{1}=\frac{p^{2}}{2 m}+\Delta^{f}(p)+\Delta^{b}(P) \\
E_{k P}=E_{k P}^{0}+\Delta_{k}^{f}(p)+\Delta_{k}^{b}(P)
\end{gathered}
$$


The essential step is now to identify the shifts for zero momentum with the chemical potentials of quasispecies (bound states)

$$
\begin{gathered}
\varepsilon_{1}=\frac{p^{2}}{2 m}+\Delta^{f}(p)+\Delta^{b}(P) \\
\mu_{2}(k)=\mu(1)+\mu(2)+E_{k}^{0}+\Delta_{k}(0)
\end{gathered}
$$

Each bound state $k$ is considered as a separate chemical species [20]. After identifying the chemical potentials for the new species one goes to the free energy density by standard methods. Finally we have to require that the free energy density is minimized with respect to the division into the different chemical species

$$
\begin{aligned}
& f\left(\beta, n_{1}, n_{2}^{(k)}, \ldots\right)=\min \\
& n=n_{1}+\sum_{k} n_{2}^{(k)}+\ldots
\end{aligned}
$$

The quasi-particle picture given here is consistent with the usual chemical picture [8]. However in different to earlier approaches now all the bound states are considered separately (compare $[5,6]$ and $[20]$ ).

\section{Thermodynamic properties of hydrogen helium systems}

Since the matter constituting the sun is a mixture of hydrogen and helium we need an expression for the energy including at least double charges and three-particle bound states. We shall use eqs. $(7-11)$ and $(16-21)$ as the theoretical basis for constructing the free energy. Let us consider now several contributions separatly. The ideal free energy density has the form (in Rydberg units) :

$$
\begin{gathered}
f_{I D}=f_{I D}^{(e)}+\tau \sum_{i} n_{i}\left[\left[\ln \left(n_{i} \Lambda_{i}^{3} / \widetilde{g_{i}}\right)-1\right]+\sum_{k} n_{k}^{(e i)}\left[E_{k}^{(e i)}+\ln \left(n_{k}^{(e i)} \Lambda_{e}^{3} \overline{g_{k}^{(e i)}}\right)-1\right]\right. \\
+\sum_{k} n_{k}^{(e e i)}\left[E_{k}^{(e e i)}+\ln \left(n_{k}^{(e e i)} \Lambda_{e e i}^{3} \widetilde{g_{k}^{(e e i)}}\right)-1\right]+\sum_{k j} n_{k}^{(e j i)}\left[E_{k}^{(e j i)}+\ln \left(n_{k}^{(e j i)} \Lambda_{e j i}^{3} \widetilde{\left(g_{k}^{(e j i)}\right.}\right)-1\right]+\ldots
\end{gathered}
$$


Here the first contribution denotes the electronic part including degeneracy and

$$
E_{k}^{(e i)}, E_{k}^{(e e i)}, E_{k}^{(e j i)}
$$

the bound state energies of two or three particles respectively not including shifts. These shifts - which are strictly speaking already effects of nonideality - are defined by eqs. (17-20).

The quantum number $k$ represents the energy states of hydrogen-like (ei), helium-like (eei) or molecular ion-like (eii) bound states. The reduced weight factor of the state $k$ with the degeneracy $g_{k}$ is according to a Brillouin-Planck-Larkinrenormalization $[13,17] \widetilde{g}_{k}$ :

$$
\tilde{g}_{k}(T)=g_{k}\left[1-\exp \left(\beta E_{k}\right)+\beta E_{k} \exp \left(\beta E_{k}\right)\right]
$$

The sums over $i$ and $j$ in eq. (22) cover only the positive particles (nuclei); $\Lambda$ is the De Broglie wave length.

All the densities refer to chemical species in the sense discussed above; they are not identical with the total densities of electrons and nuclei.

For the Coulombic part we have constructed in earlier work several Padé approximations which comprise the available analytical knowledge [1 8-22].

A special property of these Padé formulea is the smooth transition from a behaviour like $\mathrm{n}^{1 / 2}$ at small densities to $\mathrm{n}^{1 / 3}$ at high densities. Padé approximations are not unique; several variants may be found elsewhere [18-21]. In earlier work applications of these formulea to hydrogen plasmas $[17,18]$, alkali plasmas [13], noble gas plasmas [19] and to electron. positron plasmas [21] were given. Here we concentrate our consideration on helium plasmas and hydrogen-helium plasmas.

In the calculations discussed below, we have used the following formula: 


$$
\begin{aligned}
& f_{C O}=f_{C O}^{(e)}-\tau n_{+} \frac{q_{0} \widetilde{n}^{1 / 2}+1000 \widehat{n}^{3 / 2} e_{1}(\tilde{n})}{1+q_{1}(\tilde{n} \tau)^{1 / 2}+1000 \widehat{n}^{3 / 2}}-\tau n_{+} \frac{Q_{0} \widetilde{n}^{1 / 2}+\bar{n}^{3 / 2} e_{2}\left(\hat{n}, r_{s}\right)}{1+Q_{1}(\tilde{n} \tau)^{1 / 2}+\bar{n}^{3 / 2}+Q_{4} \widetilde{n}^{1 / 2} \ln \left(1+Q_{5} \tilde{n}^{1 / 2}\right)} \\
& q_{o}=1.1816\left(\mathrm{z}^{2}\right)^{3 / 2} ; \zeta_{i}=n_{i} / n_{+} ; \gamma_{I}=m_{e} / m_{i} \\
& q_{0} q_{1}=0.696 \sum_{i j}^{+} \zeta_{i} \zeta_{j} z_{i}^{2} z_{j}^{2}\left(\gamma_{i}+\gamma_{j}\right)^{1 / 2} \\
& e_{1}(x)=1.447 x^{1 / 3}-4.2944 x^{1 / 12}+0.6712 x^{-1 / 12}+0.2726 \ln x+2.983 \\
& \zeta_{e}=n_{e} / n_{+} ; \bar{n}=n_{e} \Lambda_{e}^{3} ; \tilde{n}=n_{+} \beta^{3} e^{6} ; \widehat{n}=\widetilde{n}\left(2^{5 / 3}\right)^{3}\langle z\rangle \\
& Q_{1}=\left[2.876 \zeta_{e}\left(z^{2}\right)-1.666 \zeta_{e}^{2}-q_{0} q_{1}\right] / Q_{0} \\
& Q_{0}=1.1816\left[\left(\left(z^{2}\right)+\zeta\right)^{3 / 2}-\left(z^{2}\right)^{3 / 2}-\zeta^{3 / 2}\right] ; \zeta=n_{e} / n_{+} \\
& Q_{3}=.5236\left(\left\langle z^{3}\right\rangle-\zeta\right)-\left\{4\left(z^{3} \ln z\right\rangle+\left(\left(z^{3}\right)-\zeta\right) \cdot \ln \left[29.09\left(\left(z^{2}\right)+\zeta\right)\right]\right\} / Q_{0} \\
& Q_{4}=.5236\left\{\left(z^{3}\right)-\zeta\right)^{2} / Q_{0} ; Q_{5}=\exp \left(-Q_{3} / Q_{4}\right) \\
& e_{2}(x, y)=\frac{.8511 y x^{1 / 2}}{\left(1+.3135 x^{-1 / 2}\right)\left(1+1.137 y \tau^{1 / 2}\right)}+\frac{y x^{1 / 3}(.0726+.0161 y)}{\left(1+.0887 y^{2}\right)} ; r_{s}=d_{e} / a_{B}
\end{aligned}
$$

\section{Ionization processes}

The free energy and the composition are obtained by minimization of the free energy density after eqs. (7), (11), (2123), (24) under the constraint that the total number of electrons is constant. Let us first consider helium plasmas. In order to describe the state of ionization we introduce several definitions

$\alpha$ - part of electrons which are free,

$\alpha_{2}$ - part of the nuclei which are free,

$\alpha_{1}$-part of the nuclei which have bound one electron,

$\alpha_{0}$ - part of the nuclei which have bound two electrons.

We consider now $\alpha$ (degree of ionization of the electrons and $\alpha_{2}$ (degree of double ionization of the nuclei as the independent quantities. The relations following from the balance for the electrons and nuclei

$$
\begin{gathered}
\alpha_{0}=1+\alpha_{2}-2 \alpha \\
\alpha_{1}=2 \alpha-2 \alpha_{2}
\end{gathered}
$$

may be used to determine the other quantities defined above. Figs.1-2 show the results obtained by minimization of the free 
energy at $\mathrm{T}=30000 \mathrm{k}$ and $\mathrm{T}=50000 \mathrm{k}$ as a function of the total density of nuclei.Looking at Figs. 1-2 we see that the degree of electron ionization $\alpha$ (number of free electrons related to the total number of electron) decreases with increasing density and after reaching a minimum increases again $\left(n_{\eta}\right.$ - total density of the nuclei). The latter effect is called pressure ionization. Beyond the minimum the density of free electrons is very high and the system will behave similar to a metal plasma. At intermediate densities of the nuclei (around $10^{22}-10^{23} \mathrm{~cm}^{-3}$ ) the free energy shows two minima what leads to two possible values of $\alpha$ and $\alpha_{2}$ at fixed nuclei density $n_{\eta}$.

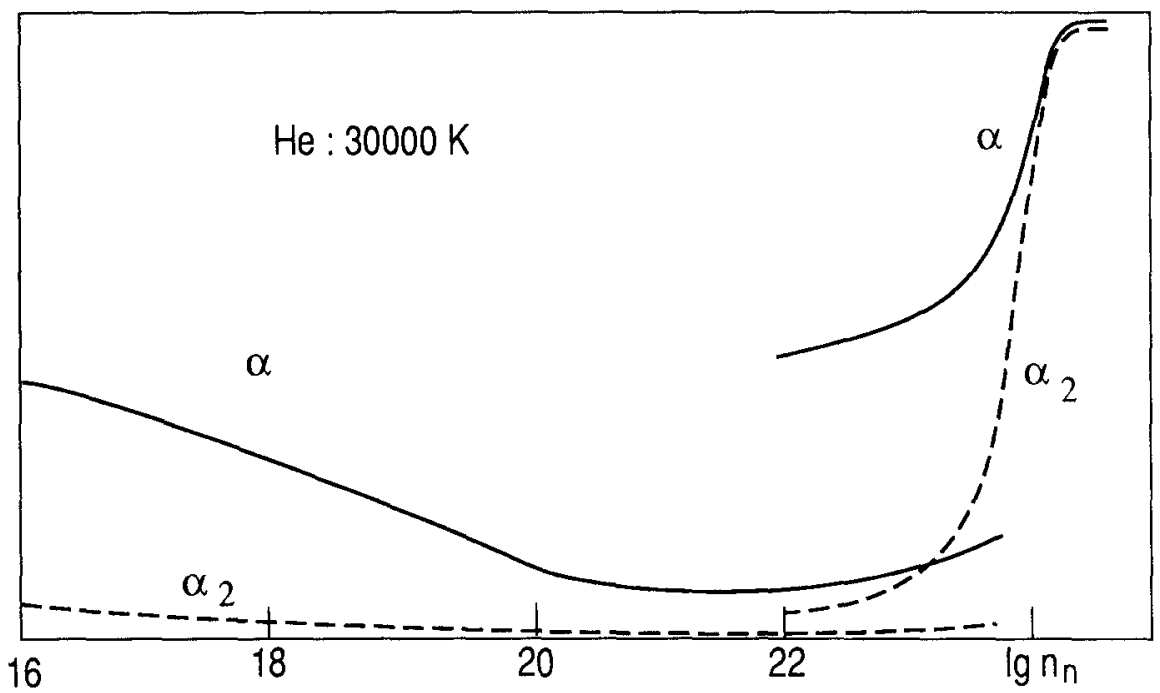

Fig. 1 Degree of ionization of the electrons $(\alpha)$ and degree of ionization for the nuclei $\left(\alpha_{2}\right)$ for He-plasmas at $T=30000 \mathrm{~K}$.

The existence of two minima of the free energy is always to be considered as a hint to phase transitions of first order. Based on the curves shown in Figs. 1-2 we would expect a plasma phase transition in helium plasmas at temperatures $T \leq T c \approx$ $55000 \mathrm{k}$. This is much higher than the estimate given by Hess [23] Tc $\approx 28500$ which was obtained by consideration of single ionization only. We see here that the possibility of double ionization favours instabilities and phase transitions. 
This tendency was observed already in an early work by Valuev, Medvedev and Norman [24]. Our consideration is more quantitative since it is based on more recent expressions for the free energy. Unfortunately even this is not yet enough for an exact calculation of the phase transition. Such a quantitative theory requires according to thermodynamics rules :

(i) Complete calculation of the free energy surface in dependence on all independent densities,

(ii) Construction of tangent planes connecting two minima which correspond to coexisting phases.

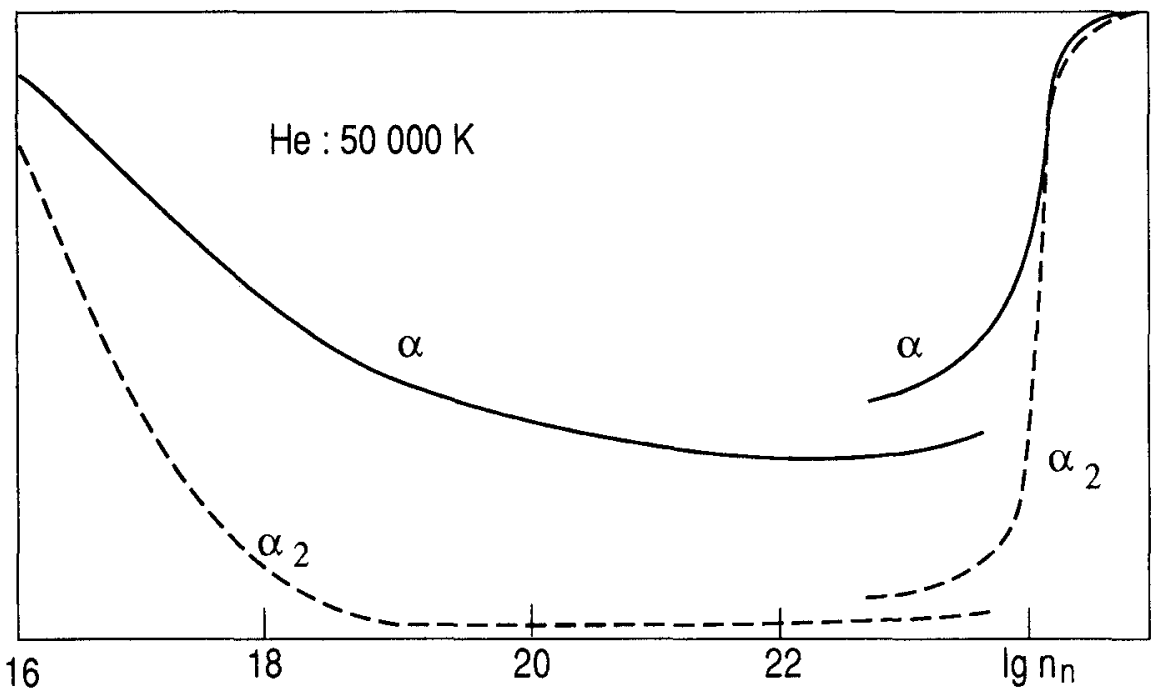

Fig. 2 Degree of ionization for He at $\mathrm{T}=50000 \mathrm{~K}$.

In our calculations given above we have assumed that the two phases coincide in the mass densities and differ only in the degrees of ionization. This is only a first approximation to the real situation where the two phases may differ also in the mass densities. 
Let us consider now mixtures of hydrogen and helium with a mole fraction of about 0.93 for the hydrogen component (Fig.3)

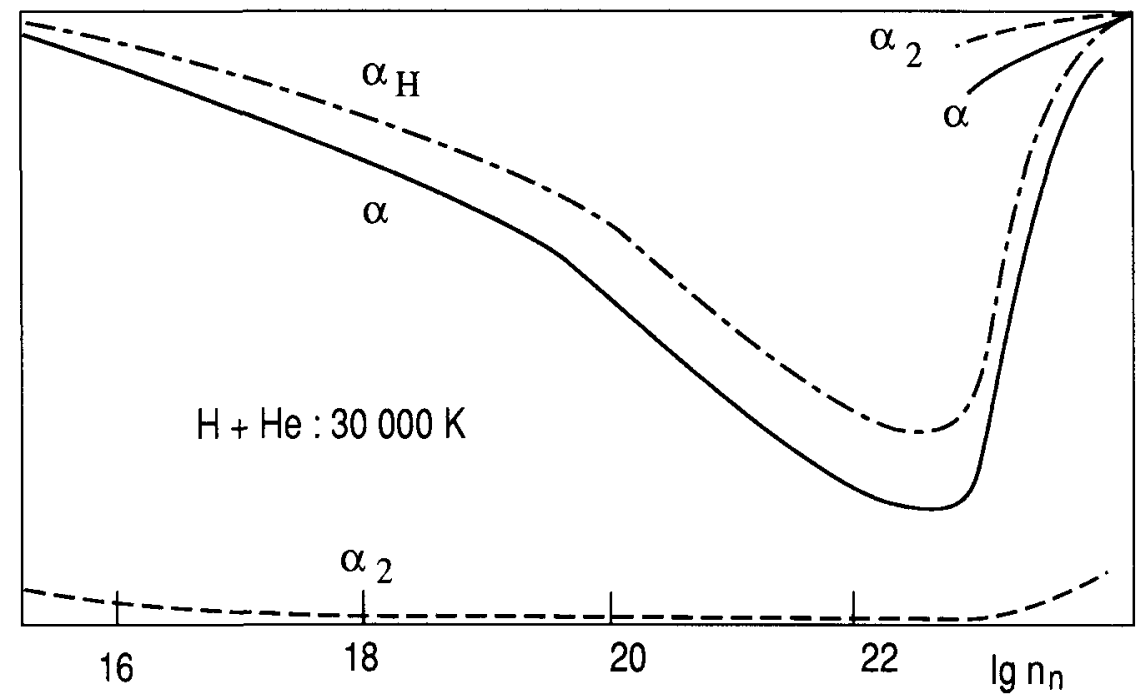

Fig.3 Degrees of ionization for a hydrogen-helium mixtures (93\% H and $7 \%$ He in mole percents).

As Fig. 3 shows, we observe again a characteristic minimum in the degree of ionization for helium $(\alpha)$ and for hydrogen $\left(\alpha_{H}\right)$ at total nucleus densities $10^{22} \cdot 10^{23} \mathrm{~cm}^{-3}$ and a strong increase of higher values. It is interesting to note that in a narrow density region $\left(10^{23} \mathrm{~cm}^{-3}\right)$ at $\mathrm{T}=30000 \mathrm{k}$ again two minima of the free energy, i.e. two values for the degree of ionization are observed. This points to the existence of a thermodynamical instability

$$
\left[\frac{\partial \ln p}{\partial \rho}\right]_{T}<0
$$

leading to a plasma phase transition in $\mathrm{H}$ - He - plasmas with a critical point of about $\mathrm{T} \approx 35000 \mathrm{k}$. Let us mention that the estimate for pure $\mathrm{H}$ - plasmas is $\mathrm{T}_{\mathrm{c}}=16500 \mathrm{k}$ [18]. Again we see, that two-fold ionization favours instabilities and phase transitions. The relative high electron density leads to a strong decrease of the ionization energy for two-fold ionization and 
makes two-fold much more favourable than one predicts on the basis of the ideal theory. Having in mind that He has a rather high second ionization energy $(54.1 \mathrm{eV})$ one may expect much stronger enhancement of two-fold ionization for the noble gas plasmas Xe $(21.2 \mathrm{eV}), \mathrm{Ne}(41.1 \mathrm{eV})$ etc. and for the earth alkaline plasmas Ca (11.9 eV), $\mathrm{Mg}(15.0 \mathrm{eV}), \mathrm{Hg}(18.8 \mathrm{eV})$ etc. Let us still mention that for a dynamical theory of stars beside the traditional instabilities as eq. (26) also other instabilities as

$$
\left[\frac{\partial \ln p}{\partial \rho}\right]_{S}<\frac{4}{3}
$$

are of fundamental interest. It remains open for further investigations to check our equation of state with respect of instabilities of the type (27).

Let us discuss now briefly the question of ionization rates following the results given in earlier papers [13,21, 26-28].

We restrict the following consideration to two-particle bound states. From eqs. (17-18) follows a close connection between the interaction parts of the chemical potentials

$$
\delta \mu_{a}=\frac{\partial\left(f-f_{I D}\right)}{\partial n_{a}}
$$

and the effective energy levels. By application to hydrogen. like bound states we get for the density-dependent energy from (18)

$$
\widetilde{E}_{K}^{(e i)}=E_{K}^{(e i)}+\delta \mu_{e}+\delta \mu_{i}+\Delta_{K}^{(e i)}
$$

From eq. (17) follows for the continuum edge

$$
\varepsilon_{1}(0)=\widetilde{E}_{\infty}^{(e i)}=0+\delta \mu_{e}+\delta \mu_{i}
$$

The difference between both expression yields the energy gap, between the continuum and the energy state $k$. The gap shift which increases with the density is given by solutions of the Bethe-Salpeter equation $[27,28]$. A sufficiently simple approximation reads 


$$
\Delta_{k}^{(e i)}=\frac{z_{i} e^{2}}{r_{D}+r_{k} / 2} ; r_{k}=\left\langle r_{k}\right.
$$

where $r_{D}$ is the Debye radius and $r_{K}$ the mean radius of the Bohr orbit in the state $k[21,28]$.

In a first approximation the ionization rates will follow an Eyring-type law, i.e. they will change exponentially with the gap. This assumption yields the following connection between the ionization rates, their ideal value and the gap shifts

$$
\alpha=\alpha_{I D} \exp \left[\frac{\Delta_{k}^{(e i)}}{\tau}\right]
$$

A stronger derivation from kinetic equations was given in [27]. The relation (32) shows that the ionization rates increase exponentially with the nonideality.

Summarizing the results given in this paper we may state that the equation of state and the ionization processes are strongly influenced by nonideality effects in the region where the nonideality parameters given by eqs. (3-4) and (6) are sufficiently large.

\section{References}

[1] W. Ebeling, A. Engel, R. Feistel, Physik der Evolutions processe, A kademie Verlag, Berlin 1989

[2] R. Feistel, W. Ebeling, Evolution of Complex Systems, Kluwer Academic Publ, Dordrecht 1989

[3] F. A. Rogers, Phys. Rev. A$\underline{3} \underline{8}$ (1988) 5007; Astrophys. J.․ㅡ므므 (1986) 723

[4]C.A. Iglesias, Invited Lecture at the IAU.Colloquium 121 and contribution to his volume

[5] W. Däppen, Y. Lebreton, F. Rogers, The Equation of State of the Solar Interior. Contr. to IAU-Colloquium 121 ,Versailles 1989

[6] D.G. Hummer, D. Mihalas, Astrophys. J. 331 (1988) 749 W. Däppen, L. Anderson, D. Mihalas, Astrophys. J. 319 (1987) 195 ;

D. Mihalas, W. Däppen, D.G. Hummer, Astrophys. J. 331

(1988) 815 
[7] W. Ebeling, W.D. Kraeft, D. Kremp, G. Röpke, Physica 140A (1986) 160;

W.D. Kraeft, W. Ebeling, D. Kremp, G Röpke, Ann. Physik $\underline{4} \underline{6}$ (1988) 429

[8] W. Ebeling, Physica 43 (1969) 293; Z 3 (1974) 573

[9] W.D. Kraeft, W. Ebeling, D. Kremp, G. Röpke, Quantum Statistics of Charged Particle Systems, Plenum Press. New York 1986

[10] M. Ross, F.H. Ree, D.A. Young, J. Chem. Phys. I2 (1983) 1487

$11]$ M. Ross, D.A. Young, Phys. Lett 11요 (1986) 463

[12] G.A. Mansouri et al., J. Chem. Phys. $\underline{5} 4$ (1971) 1523

[13] W. Ebeling, A. Förster, R. Redmer, T. Rother, A. Schlanges, Instabilities and Phase Transitions in Dense Hydrogen, Rare Gas and Alkali Plasmas, ICPIG-18, Invited Papers, Swansea 1987.

[14] S. Galam, J.P. Hansen, Phys. Rev. A1ㄴ (1976) 816

[15] H.E. Dewitt, Phys. Rev. A14 (1976) 129

[16] R. Redmer, G. Röpke, Physica 130A (1985) 523

[17] W. Ebeling, W.D. Kraeft, D. Kremp, Bound States and Ionization Equilibrium in Plasmas and Solids, AkademieVerlag, Berlin 1976

[18] W. Ebeling, W. Richert, Phys. Lett 10으스 (1985) 80; phys. stat. sol. (b) $12 \underline{8}$ (1985) 467

[19] W. Ebeling, A. Förster, W. Richert, H. Hess, Physica 150A (1988) 159

[20] W. Ebeling, Z. phys. Chem. (Leipzig) $2 Z 11$ (1990) N ${ }^{\circ} 2$

[21] W. Ebeling, Contr. Plasmas Phys. 29 (1989) 165

[22] W. Ebeling, H. Lehmann, Ann. Physik 445_(1988) 529

[23] H. Hess, High Pressure Research 1 (1989) 203

[24] A.A. Valuev, I.T. Medvedev, G.E. Norman, Zh. eksp.teor. Fiz. (USSR) 52 (1970) 2228

[25] W.M. Tscharnuter, Instabilities in the Early Protosun Contr. to IAU-Colloquium 121, Versailles 1989; Formation of Viscous Protostellar Accretion Disks. Contr. to Conf. Accretion Disks, Garching 1989. 
[26] M. Schlanges, Th. Bornath, D. Kremp, Phys. Rev. A$\underline{3} \underline{8}$ (1988) 2174

[27] K. Kilimann, W.D. Kraeft, D. Kremp, Phys. Let t. $\underline{\text { A }} \underline{1}$ (1977) 393

[28] W. Ebeling, K. Kilimann, Z. Naturf. 44늑 (1989) 